\title{
Correction to: Adaptive Compensation of Nonlinear Actuators for Flight Control Applications
}

\section{Correction to:}

D. Deb et al., Adaptive Compensation of Nonlinear Actuators for Flight Control Applications, Studies in Systems, Decision and Control 386, https://doi.org/10.1007/978-981-16-4161-9

The original version of the book was inadvertently published with an incorrect affiliation of the editor Dipankar Deb which has now been corrected as Institute of Infrastructure, Technology, Research and Management. The book has been updated with the change. 